\title{
Statin prescribing according to gender, age and indication: What about the benefit-risk balance?
}

Helle Wallach-Kildemoes MA MPH PhD ${ }^{1 *}$, Henrik Stovring MSc PhD², Ebba Holme Hansen MSc ${ }^{3}$, Kenneth Howse BPhil $^{4}$, Hálfdán Pétursson MD PhD 5

${ }^{1}$ Associate Professor, Section for Social and Clinical Pharmacy, Department of Pharmacy, Faculty of Health and Medical Science, University of Copenhagen, Denmark

${ }^{2}$ Associate Professor, Section for Biostatistics, Department of Public Health, University of Aarhus, Denmark

${ }^{3}$ Professor, Section for Social and Clinical Pharmacy, University of Copenhagen

${ }^{4}$ Senior Research Fellow, Oxford Institute of Population Ageing, University of Oxford, UK

${ }^{5}$ Research Fellow, General Practice Research Unit, Department of Public Health and General Practice, Norwegian University of Science and Technology, Norway

\section{Correspondence*}

Helle Wallach-Kildemoes, Associate Professor,

Section for Social and Clinical Pharmacy,

Department of Pharmacy, Faculty of Health and Medical Science,

University of Copenhagen

Universitetsparken 2, 2100 Copenhagen, Denmark

Phone +4535336475

helle.wallach@sund.ku.dk

\section{Keywords:}

Statin prescribing, guidelines, indication, gender, age, benefit-risk balance

\section{Running head:}

Statin prescribing according to gender, age and indication

Word count: 4968

Tables and figures: Two Tables, four Figures 


\begin{abstract}
Rationales and aim

The increasing dispensing of statins has raised concern about the appropriateness of prescribing to various population groups. We aimed to 1 ) investigate incident and prevalent statin prescribing according to indication, gender and age; 2 ) relate prescribing patterns to evidence on beneficial and adverse effects.
\end{abstract}

\title{
Methods
}

A cohort of Danish inhabitants ( $N=4,424,818$ ) was followed in nationwide registries for dispensed statin prescriptions and hospital discharge information. We calculated incidence rates (2005-2009), prevalence trends (2000-2010) and absolute numbers of statin-users (incident and prevalent users in 2010), according to register-proxies for indication, gender and age.

\section{Results}

In 2010 the prevalence became highest for ages $75-84$ and was higher in men than women (37\% and 33\%, respectively). Indication-specific incidences and prevalences peaked at ages around 65-70, but in myocardial infarction the prevalence was about $80 \%$ in the age span 45-80. Particularly, incidences tended to be lower in women until ages about 60 where after gender differences were negligible. In asymptomatic individuals (hypercholesterolemia, presumably only indication) aged 50+ dispensing was highest in women. The fraction of statin dispensing for primary prevention decreased with age: higher for incident than prevalent prescribing. Independent of age this fraction was highest among women e.g. $60 \%$ vs. $45 \%$ at ages $55-64$. The fraction for potential atherosclerotic condition (PAC, e.g. heart failure) increased with age.

\section{Conclusion}

Prevalence of statin utilization was highest for ages 75-84, although indication-specific measures were relatively low. Despite inconclusive evidence for a favourable risk-benefit balance, statin prescribing was high among people aged $80+$, asymptomatic women, and PAC patients. 


\section{Introduction}

In 1994, statins (HMG-CoA reductase inhibitors) were introduced on the market as lipid-lowering drugs for reduction of mortality after myocardial infarction (MI) in middle-aged men with hypercholesterolemia [1]. Subsequently, recommendations for statin prescribing have gradually expanded [2] to include patients with different categories of atherosclerotic cardiovascular diseases (CVD), diabetes and, in addition, individuals assessed as being at high risk of developing CVD $[3,4]$. Today, statins are among the most prescribed medications globally, but the widespread use has provoked debate on the appropriateness of the high consumption [5,6]. An important concern is the statin use in groups of people for whom the therapeutic benefits may not outweigh adverse effects [7-9], i.e. an unfavourable benefit-risk balance. Further, it has even been questioned whether lipid-lowering guidelines are truly evidencebased $[10,11]$. The key issues in the debate are the appropriateness of statin prescribing for individuals with neither CVD nor diabetes, particularly women [12-15], and prescribing for older persons $[9,16,17]$.

Currently, most guidelines recommend treatment decisions in primary CVD prevention to be based on a combined risk estimate, rather than single risk factors [18]. Guidelines for the high risk strategy of primary CVD prevention include easy-to-use risk calculators to be used for individuals with neither CVD nor diabetes. Typically these algorithms include the risk factors age, gender, smoking status, blood pressure, and serum cholesterol. Studies on various populations have indicated that these risk calculators tend to overestimate risk $[15,19,20]$, labelling substantial proportions of generally healthy populations as being 'at risk' and in need of preventive intervention, e.g., statin treatment. However, the evidence for the effect of statins in primary prevention is equivocal, since the trials addressing the question declare to exclude individuals with pre-existing CVD, but have included heterogeneous study populations. Only few randomized clinical trials (RCTs) have excluded participants with diabetes [21] (generally regarded as CVD equivalent), whereas the majority has not [22-24]. In the following the term "primary prevention" will be applied when referring to individuals without CVD or diabetes.

Statins for secondary prevention are recommended in different categories of CVD, e.g. MI, ischemic heart disease without prior MI (IHD), ischemic stroke and peripheral arterial ischemic disease (PAD), but also potential atherosclerotic conditions (PAC) such as heart failure, aorta aneurysm and chronic kidney disease. However, the beneficial effect of statins for individuals with chronic kidney disease and heart failure has been questioned $[25,26]$. While women and older people tend to be underrepresented in RCTs, meta-analyses and literature-reviews report conflicting results regarding the beneficial effect of statins in primary prevention - particularly in women [12,21$23,27-29]$ as well as on the beneficial effect in older people in general $[16,17,30]$. As adverse effects such as muscle problems are particularly common among women and older persons [31], the benefit-risk balance most likely varies according to indication, gender and age.

Several observational studies have shown marked gender and age differences in statin utilization [32-34]. Studies focusing on primary prevention report conflicting results as to gender differences, some reporting higher use in women [33,35] others no gender differences [34]. These observational studies either include [34] or exclude [33,35] individuals with diabetes, which partly may explain discrepancies. Lower prevalence of statin prescribing in older people with CVD compared with younger has been observed and termed a treatment-risk paradox due to higher CVD risk in older ages [32,33]. Whether it truly is a paradox, however, depends on the beneficial effect of statins for older people with specific indications, as well as the age limit for categorizing individuals as old. The potential cumulative net effect over time may also be greater for the younger population.

The above described ambiguities may reflect methodological challenges in defining the target population when exploring statin utilization, both as to primary and secondary prevention. Hence, more detailed exploration of prescribing patterns according to gender, age and indication is needed to identify potentially inappropriate prescribing patterns, i.e. statin utilization in groups for whom the benefit-risk balance seems to be unfavourable. 
We have previously developed a method for register-based proxies for the indication for statin prescribing regarding new users (incident users) as well as continuing users (prevalent users) [36].

By means of the nationwide Danish registers and the register-based proxies for statin prescribing indication [36], we have shown that statin therapy is increasingly initiated among older persons and individuals with neither CVD nor diabetes [37] and that the pattern of statin utilization according to indication, gender and age differs between prevalent and incident statin users [36]. However, to assess whether the high incidence and prevalence of statin therapy is appropriate, statin prescribing patterns must be related to evidence about the beneficial effects of therapy versus adverse effects.

The aim of this study was to 1 ) investigate incident and prevalent statin prescribing according to indication, gender and age; 2 ) relate the observed prescribing pattern to the available evidence on beneficial and adverse effects.

\section{Data and methods}

A closed cohort corresponding to all Danish residents as of January 1, 1996 ( $N=5,110,128$, after excluding individuals not fully observable in 1995, cf. [36]) was followed in the Danish nationwide individual-level registries covering information on dispensed prescriptions, hospital discharges as well as date of death or emigration [38]. We retrieved data on incident and prevalent statin prescribing according to gender, age and indication (register-proxies) during 2005-2009. The cohort was, in addition, followed during 2000-2009 for trends in prevalent statin prescribing according to gender and age. To ensure the same period of historic register information, cohort members were censored in case of emigration (temporary or permanent), resulting in 4,791,618 cohort members in 2000;4,424,818 in 2005; and 4,157,682 in 2009. Table 1 shows the ageing of the closed cohort. Analyses were each year restricted to individuals aged $40+$.

Information on dispensed prescription medications was obtained from the Danish National Prescription Registry (DNPR), which holds information on all out-of-hospital prescription medicines dispensed at Danish pharmacies since 1995 [39]. Records include a person identifier, date of dispensing and the Anatomical Therapeutic Chemical classification code [40]. In line with other dispensing registries, DNPR does not provide information on prescribing indication that is readily usable for research [39].

We retrieved in-hospital information on patient discharges from non-psychiatric hospitals since 1977 from the Danish National Patient Registry [41]. Records include the admission and discharge dates, discharge diagnoses according to the International Classification of Diseases 10th revision along with codes for diagnostic and surgical procedures. Data were linked by means of a unique encrypted person-identifier [38]. Register-based studies in Denmark do not require specific approval by an ethics board [38].

\section{Methods}

To investigate statin prescribing according to indication, gender and age, we applied a previously developed indication hierarchy [36] based on historical and actual register-indicators (in-patient and/or prescription information) on eight mutually exclusive medical indications for statin prescribing. The indication hierarchy (Table 2) largely follows statin recommendations described in Danish and European guidelines for CVD prevention [3,4] and includes in hierarchical order: MI; IHD; ischemic stroke; PAD; PAC; diabetes; primary uncomplicated hypertension; together with a 'no-diagnosis' group without any of the aforementioned register-markers, i.e. presumably mainly individuals with elevated cholesterol-levels as the only indication. Individuals with primary hypertension or nodiagnosis (referred to as asymptomatic individuals) correspond to the target group for the high-risk strategy in the primary prevention of CVD. The PAC group was included to avoid misclassifying those often seriously ill individuals as asymptomatic individuals, and to get insight into statin prescribing for this grey zone indication.

By means of the indication hierarchy [36], all cohort members were continuously assigned to the indication for potential statin prescribing with highest rank. Prevalent statin-users were defined as individuals who by January 1 
(index date) had at least one statin dispensing during the preceding 365 days, and incident statin-users were defined as non-prevalent users with their first statin dispensing within 365 days after the index date. The indication among incident users was defined as the indication level reached at the date of first dispensing, whereas the indication among prevalent users was defined as the indication level reached at the index date for the current year.

Stratified by indication, gender and five-year age groups, we calculated the mean absolute number of incident statinusers as the annual average for the years 2005-2009, and the number of prevalent users as of January 1, 2010. The mean yearly treatment incidence rate during 2005-2009 was defined as the yearly number of stratum specific incident statin-users relative to the person-years at risk, averaged over the period - with right censoring at death, emigration or transition to a higher indication level, whichever came first. Treatment prevalence according to indication, gender and age (the point prevalence proportion) was calculated as the number of statin-users at the index date in 2010 per 100 cohort members at the same date. The overall trend in statin treatment point prevalence during 2000-2010 was calculated according to gender and age. Age was defined as the age at the beginning of each calendar year.

All analyses were performed using Stata Version 13.1 (StataCorp, College Station, TX, USA).

\section{Results}

Figure 1 shows the increase in overall statin treatment prevalence during 2000-2010 according to gender and age. While the prevalence was slightly higher among men than women for all ages, the increase in prevalence over time was most pronounced among ages above 75 . At the end of the decade the prevalence among 75-84 year olds had become the highest, 38/100 and 32/100, for men and women, respectively The steep increase among those aged $85+$ began at a later point in time than in other age-groups, but reached a similar level as for those aged 55-64 years, $22 \%$ and $18 \%$, for men and women, respectively

Figure 2 shows the indication-specific incidence rate (2005-2009) and point prevalence (January 2010) of statin prescribing, according to gender and age - for MI, IHD, stroke and PAD. While the incidence of statin prescribing for IHD, stroke and PAD peaked around the age of 60 years the prevalence peaked about ten years later. For MI, the incidence of statin prescribing decreased with age, while the prevalence reached $75-80 \%$ independently of gender and age until the age 75 . For all indications, but most pronounced for $\mathrm{MI}$, the incidence of prescribing at ages below 65 was lower in women than men. The differences were less pronounced regarding prevalent prescribing.

Figure 3 shows - in analogy to Figure 2 - indication-specific incidence rates and point prevalences of statin prescribing according to gender and age, as to the indications PAC, diabetes, hypertension and no-diagnosis. The incidence of statin dispensing peaked around the age of 65, and less accentuated and with some delay for prevalent dispensing. For diabetes, but also for PAC and hypertension, a higher incidence of statin dispensing was observed at ages below 60 years among men than women, after this age the opposite tended to be the case. The gender differences at ages below 60 narrowed among prevalent users, and the prescribing in diabetes peaked at ages 65-70 with a prevalence of around $60 \%$ for both genders. For the no-diagnosis category both incidence and prevalence of statin therapy among individuals aged 50+ was considerably higher in women than in men.

Figure 4 shows the indication-specific proportion of yearly number of incident statin-users (averaged over 20052009) according to gender and age, compared to the corresponding figures for prevalent statin-users by January 1 , 2010. For both incident and prevalent use, the proportion of statin-users without pre-existing CVD or diabetes decreased with age. Independent of age, this proportion was higher in women than men. Among incident users aged 55-64 60\% of female users were asymptomatic and $46 \%$ among male users; at ages $65-74$ the corresponding figures were $50 \%$ and $35 \%$, respectively. For prevalent use a similar gender related pattern was observed, but with a higher proportion of users with pre-existing MI or IHD. In ages above 75 , a relative high proportion of prevalent statin-users 
had either PAC or ischemic stroke as indication, corresponding for ages $75-84$ to $31 \%(17 \%+14 \%)$ and $25 \%$ $(11 \%+14 \%)$ among women and men, respectively.

\section{Discussion}

\section{Main findings}

In this nationwide Danish cohort study the prevalence of statin therapy increased substantially between 2000 and 2010 , and had become the highest among individuals aged 75-84 by the end of observation period (2010). Throughout the period the prevalence was highest in men. For most indications, indication-specific incidences and prevalences peaked at younger ages, around 65-70. For patients with myocardial infarction, in contrast, the prevalence of therapy was about $80 \%$ over the age range $45-80$.

For ages below 60 statin prescribing, in particular incident prescribing, tended to be lower in women than men, but after this age gender differences were negligible. In contrast, incidences as well as prevalences of therapy among individuals aged $50+$ in the no-diagnosis category were considerably higher in women than men. While the proportion of statin-users in primary prevention decreased with age, this proportion was highest for incident therapy and higher in women than men, e.g. at age 55-64: 60\% and 45\% in women and men, respectively. The fraction with PAC increased with age. More than $25 \%$ of prevalent statin-users aged $75+$ had PAC or ischemic stroke as indication for statin therapy, the fraction being higher in women than men.

\section{Strengths and limitations}

We consider it a major strength being able to follow an unselected Danish cohort prospectively for statin dispensing in the nationwide individual-level registries. As the study is based on nationwide registers, the loss to follow-up is very limited. Selection bias may still be present though, as we excluded those not fully observable in the ten years preceding the study period, corresponding to presumably healthier young individuals who reside temporarily abroad and immigrants (about $4.3 \%$ of the population). This under-representation may have resulted in a slight overestimation of prevalence and incidence of statin prescribing - especially in younger age-groups, and a general under-representation of immigrants. Our end of follow-up date at December 2009 may be regarded a limitation. However, aggregate information on dispensed prescription medicines reveals that the prevalence of statin therapy at ages above 65 continues to increase. As Danish recommendations (following the European) to a large extent have remained unchanged, we believe that our results - and concerns - reflect both Danish prescribing patterns of today and prescribing patterns in other European countries.

Another strength of the study is that by means of a previously developed register-based indication hierarchy [36], we were able to distinguish between several possible indications for statin prescribing. We do, however, recognize the possibility of misclassification, e.g. individuals may not be scored as high in the hierarchy as they should be, especially for conditions not leading to hospitalization such as individuals with IHD treated initially without hospitalization (cf. [36]).The inclusion of the PAC group is considered a major strength, because individuals with these potential atherosclerotic conditions would have been misclassified downwards in the hierarchy to the primary prevention group - and also because statins may be prescribed for these conditions despite inconclusive evidence for their beneficial effect. One major limitation of the study is the lack of information on familial hypercholesterolemia, potentially allocating the approximately 10,000 individuals with familial hypercholesterolemia in Denmark [42] at the no-diagnosis level.

The strict use of pharmacoepidemiological definitions adds to the robustness of the study design: We censored individuals at death/emigration and allowed for a shift to a higher indication level when estimating statin incidence rate according to indication, gender and age. Finally, by exploring both incident and prevalent statin prescribing we were able to unveil indicators for prescribing behaviour (incidence), as well as for continued dispensing behaviour (prevalence). However, applying cross-sectional measures to compare yearly incident statin use during 2005-2009 with point prevalence of statin use in 2010, we have introduced skewed age comparison, as prevalent statin users in 
2010, are compared to incident statin users of the same age - three calendar years (on average) before. Provided a reasonably stable incident statin prescribing for each indication according to 5-years age groups during 2005-2009, the distribution of continuing statin users should - all other things being equal - shift towards higher ages as our findings also demonstrate (reflecting the ageing of the cohort). A prospective design following incident statin users over time is required to explore whether 'upgrade' to a more severe indication and/or differences in discontinuation/ mortality rates may explain the finding that the proportion of statin therapy for primary prevention is higher among incident than prevalent users.

\section{Comparison with other studies}

In line with earlier studies on statin utilization $[32,43]$, we found a bell-shaped pattern of statin utilization according to age for most indications. However, our study demonstrated that this decrease happened at younger ages for incident than for prevalent therapy ( $60 \mathrm{vs.} 70$ years), most likely explained by the ageing of the incident users and low discontinuation rates. In accordance with a recent study from USA [44], we found that the overall prevalence of statin prescribing was highest among people aged 75-84. These seemingly contrasting findings in our study may be explained by the fact that most statin prescribing indications are prevalent in older people, i.e. there is a range of options for prescribing statins in each individual. In line with a Finnish prevalence study (population aged 70+) [33], our study demonstrated that statin therapy for older people primarily was prescribed for secondary prevention, particularly as to men and prevalent prescribing. The latter is most likely explained by higher statin discontinuation rates in primary prevention than in secondary prevention. Compared to our findings from 2005 [36] the fraction of incident statin prescribing for primary prevention has increased (for women aged $65-74$ from $50 \%$ in to an average of 55\% during 2005-2009), while the total number of incident users has decreased, indicating that most prevalent CVD or diabetes patients already are in statin therapy. On the other hand, the point prevalence of statin utilization has almost doubled during 2005-2010, with the most marked increases in the oldest segment - most likely explained by the ageing of the cohort of statin users combined with disequilibrium between the numbers of incident users and discontinuing/decedent users [45].

Confirming results from other studies [46-48], we found that statin utilization in individuals with CVD or diabetes was lower in women than in men. However, we found that these gender differences exclusively occurred at ages below 60 and particularly for incident prescribing, which may indicate lower discontinuation rates in women than men. The fact that no differences were observed after the age of 60 may further indicate that statins tend to be prescribed according to lipid-levels (which tend to be low in women before menopause), but according to recommendation for secondary prevention and diabetes statin therapy should be initiated independent of lipid-level (REF). In contrast, among MI patients we found that the prevalence of statin therapy was almost independent of gender and age until the age of 80 , although the incidence of therapy peaked approximately at the age of 50 , which may be explained by the high and age-related increase in mortality among MI-patient (both those with first $\mathrm{Ml}$ and prior $\mathrm{MI}$ ) before initiating statin therapy as out-patients, included with PYR in the denominator. Moreover, especially older individuals are most likely included as prior MI-patients. Our study revealed, further, that a relatively high proportion of prevalent statin-users aged above 75 seemed to have either ischemic stroke or PAC as indication (about 25\% and $30 \%$ in males and females, respectively). This is of concern because the evidence for statin's beneficial effect for patients with pre-existing ischemic stroke is increasingly debated [49-51], and because the evidence for beneficial effect in seriously ill patients with PAC such as heart failure [25] and advanced kidney disease [26] is inconclusive.

Studies from other Scandinavian countries $[33,47]$ with primary prevention strategies similar to the Danish have found that women with neither CVD nor diabetes are more likely to be prescribed statins than men, despite the lower CVD risk in women. This is in line with our study, but we found that this gender related treatment-risk paradox primarily holds for asymptomatic individuals without hypertension, i.e. presumably with elevated cholesterol as only indication. This paradoxical prescribing pattern is most likely a consequence of guidelines recommendation [4], where 'high' cholesterol levels, rather than high combined CVD risk, in practice, is often the reason for statin prescribing among women without hypertension at age 50+. An analogous gender-age pattern was observed among 
individuals with diabetes or CVD, indicating a strong focus on cholesterol-levels when prescribing statins, despite the fact that statins in these groups are recommend irrespective of cholesterol levels.

In contrast to our study, Sheppard et al. [34] found virtually no gender difference in the prevalence of statin therapy for 'primary prevention' in the UK. Moreover, the age-related decline in prescribing of statins for primary prevention was observed later in UK than in Denmark [34]. Apart from potential unequal discontinuation rates, the described discrepancies may have several explanations. First, the two studies apply different source populations, since Sheppard et al. included diabetic individuals in their analysis in contrast to our analysis. Second, the strategy for CVD risk screening differs between the two countries. In Denmark, the high-risk strategy to prevent CVD (primary prevention) has been implemented through opportunistic screening for high CVD risk, primarily at the GP's office [4], while the National Health Service promotes a universal screening program, inviting every UK citizen without CVD age 40-74 to a health check every five years [52]. Third, risk scoring algorithms differ between the two countries. The algorithm recommended by Danish guidelines [4] is validated for ages 40-65. British guidelines recommend the QRISK2 algorithm [53], for individuals aged 40-74. These algorithms differ regarding risk factors included.

\section{Prescribing patterns, guidelines and available evidence}

The dramatic increase in statin prescribing in both gender and all ages - but especially among older adults and women - may have several explanations: Guideline updates broadening indications and lowering cholesterol goals; promotion by stakeholders including the pharmaceutical industry; lower statin prices; GPs' preference for medication, to name a few potential causes. Obviously, clinical guidelines affect statin prescribing patterns, underlining the necessity of high quality guidelines that translate all relevant evidence into best clinical practice. This may, however, not always be the case.

While the evidence for statins for individuals with neither CVD nor diabetes, i.e. primary prevention, continues to be debated [21,24,54-56], the evidence supporting statin use for patients with MI/IHD, PAD and diabetes is strong [1,57-59]. However, the evidence supporting statin prescribing for secondary prevention in patients with ischemic stroke $[49,60]$ as well as patients in the PAC group, e.g. advanced kidney disease [26], aorta aneurism [57,61] and heart failure [25,62], is challenged.

Currently, most guidelines recommend decisions on statins for primary prevention to be based on estimated risk of fatal or any CVD event (usually over 10 years), but national guidelines recommend different risk scoring algorithms and different thresholds for high CVD risk [63], which partly may reflect calibration of risk calculators to the actual target population. However well-calibrated, several of the widely used risk algorithms, including the European SCORE [64], seem to overestimate CVD risk in the target population considerably - especially in women $[11,15,65]$. For example, it has been shown that the risk calculator published by the American College of Cardiology (ACC) and the American Heart Association (AHA) in 2013 [66] overestimate risk by as much as $150 \%$ in large US populations $[19,67,68]$. Overestimation of risk may partly stem from most risk calculators' assumption of linearity of the association between risk factors and CVD events - independent of gender and age - although many epidemiological studies indicate non-linear associations $[15,16,69]$, as well as gender and age differences $[15,16,70]$.

Recently, risk thresholds for initiation statin therapy in primary prevention have been lowered considerably in the US [66] as well as the UK [71], leading to a greatly increased proportion of the population becoming eligible for statin therapy. Interestingly, while European guidelines [72], including the Danish [4], still recommend specific lipid goals for statin treatment, the 2013 AAC/AHA guidelines have abandoned these goals [66], and the 2014 NICE guidelines [71] recommend a proportional decrease (40\%) in non-HDL cholesterol as a target rather than any specific value. Moreover, the recommendations of statins for primary preventions are based on RCTs including participants with diabetes [24]. Thus, the recommendations' evidence-base may be biased in favour of statin treatment.

The above may reflect that translating relevant evidence to practice in clinical guidelines covers recording and interpreting/analysing data, weighting results (benefits versus adverse effects) and choice of action [73]. Thus, the 
risk thresholds for initiating statin therapy in primary prevention is somewhat arbitrary and, in the end, represent subjective choices made by the guideline committees - apparently without taking sufficiently into account the consequences of lowering cut-offs: an increasing number of statin-users for whom the benefit-risk balance may be unfavourable; and the workload imposed on the healthcare systems.

\section{Statin prescribing in women and older adults: A favourable benefit-risk balance?}

The increasing statin prescribing in asymptomatic women (with neither CVD nor diabetes) and in older adults in general warrants special attention as to the benefit-risk balance.

The efficacy of statins for asymptomatic women tends to be assessed in meta-analyses without stratifying by gender $[14,24,55,74]$ and to be reported in relative terms. In fact gender-stratified meta-analyses on statin therapy for primary prevention indicate less or non-existent decrease in CVD events (non-existent as to mortality) among women, compared with men, and the evidence remains inconclusive $[21,22,27,29,75]$. While most studies include participants based on CVD history and cholesterol fractions one study (merely 21 months follow-up) apply high Creactive protein as inclusion criteria [21], indicating the same effect in men and women. Adding to this, most RCTs on statins for primary prevention do not exclude participants with diabetes. Hence, the evidence for the beneficial effect of statin therapy, especially in hypercholesterolemic women with neither CVD nor diabetes, is inconclusive.

Conclusions on meta-analyses/reviews on statins for older adults (aged $65-74 ; 75-84 ; 80+$ ) are conflicting $[16,17,30,76]$, potentially reflecting difference in inclusion criteria and end-points. In fact, guidelines on statins in old age are vague [4,9]. For older adults without CVD aged 60+ statins seem to reduce all-cause and CVD mortality [17] but this may be driven by the effect among the 'young-olds' (65-74). Analyses limited to individuals aged 80+ reveal no convincing evidence for the life-time gaining effect of statins in individuals with CVD $[9,16]$ - and certainly not among those without CVD [30]. The evidence is also inconclusive regarding statins for preventing ischemic stroke in old ages [77,78]. A literature review from 2010 concluded: "There is not sufficient data to recommend anything regarding initiation or continuation of lipid-lowering treatment for the population aged 80+, with known CVD, and it is even possible that statins may increase all-cause mortality in this group of elderly individuals without CVD" [16].

Statin related adverse reactions such as muscle problems and diabetes have long been documented and debated [31,79-83]. The biological pathway for muscle weakness is well-known [84]: Statins deplete co-enzyme Q10, leading to mitochondria dysfunction, and consequently muscle pain and weakness - demonstrated in a study on young welltrained men [85]. Although especially 'non-serious' adverse effects are underreported in RCTs [80,81], both muscle related adverse effects and unset of diabetes seem to be more frequent in women [86,87] and older adults [88]. Hence, the high statin utilization in old age is not merely of concern due to the inconclusive evidence on the beneficial effect but especially due to drug-to-drug interactions and adverse effects such as muscle weakness which most likely reduce mobility and physical activity - essential for maintaining good overall health in late life [89]. In fact, muscle problems seem to be a predictor for statin discontinuation [90], and as abrupt withdrawal of statin therapy may introduce an inflammatory rebound effect [91-93], there is a need for recommendations on how to discontinue statin therapy.

The above suggest that the benefit-risk balance often will be unfavourable in women prescribed statin for primary prevention and for the majority of people aged $75+$. Various factors may explain our finding that statin prescribing for primary prevention is more likely in women than men, despite their lower CVD risk. First, cholesterol levels increase among women after menopause [94]. Second, women may be more prone to seek health care than men at same age; and third, physician may prescribe preventive statin therapy in asymptomatic women based merely on 'high' cholesterol levels, which seems to be an overestimated CVD risk factor in women [15]. Fourth, it has become a conventional wisdom that 'high' cholesterol levels should be lowered to prevent CVD. 
The increasing statin prescribing for people aged 75+ may reflect insufficient revision of long-term prescribing that may no longer be appropriate as well as prescribing for indication with inconclusive evidence, e.g. for primary prevention and in patients with stroke/transient cerebral ischemic attack, heart failure and chronic kidney disease (PAC).

\section{Policy perspectives}

The trends in statin prescribing raise several issues for health policy, and they all have an ethical dimension. First, is the current 'high-risk' approach the most appropriate strategy for primary prevention of CVD? Second, provided the strategy makes sense at the individual level, is the allocation of resources in primary care for the strategy equitable? Third, is widespread statin prescribing in old age in the best interest of the older adults $(+75)$ ?

As Geoffrey Rose pointed out some 30 years ago, there are two strategies of prevention [95]: The high-risk strategy aims at identifying those individuals at highest risk of suffering from a disease and who will benefit from preventive measures to lower their risk, whereas the population strategy aims to lower exposure to risk factors (e.g. by promoting physical activity and reducing exposure to tobacco on a population basis). Constantly lowering the CVD risk thresholds for initiating statin therapy in the high-risk strategy to prevent CVD implies an ever decreasing clinical benefit and ever less favourable benefit-risk balance in otherwise healthy people. Adding to this, the high-risk strategy to prevent CVD gradually becomes very resource demanding as it captures increasingly larger proportions of the population among whom the benefit-risk balance may be unfavourable for many and even harmful for some individuals.

Allocating scarce resources in primary care for health checks and CVD preventive controls will inevitably leave less resources for individuals with experienced health problems, e.g. among the increasing proportion of older people in ageing societies - especially the socially disadvantaged. For policy decisions statin-therapy in older adults should be evaluated in a gerontological perspective and in terms of appropriateness, i.e. adverse-beneficial effect ratio, rather than in a single disease perspective: Having exceeded the population average lifespan, the best interest of older adults may be to allocate scarce resources on interventions that add life to years - rather than interventions aiming to add years to life [96].

\section{Conclusion}

This large Danish register study revealed a dramatic increase in statin utilization during the study period, 2000-2009, especially among the elderly (aged $75+$ ). Notable was also the higher proportion of statin prescribing for primary prevention among women than men.

In light of available evidence on both beneficial and harmful effects, the observed statin prescription pattern is of some concern. The benefit-risk balance seems to be tipped unfavourably for a considerable proportion of older individuals (for primary, as well as certain inconclusive indications of secondary prevention), as well as for women in primary prevention. While the high prevalence of statin prescribing for older people may both reflect low discontinuation rates and, perhaps, an overestimation of the net benefit in old age the high prevalence of statin therapy in asymptomatic postmenopausal women may be a consequence of the increasing cholesterol levels around menopause, although the evidence supporting statin therapy in this group is inconclusive. A clear policy and updated evidence-based recommendations on statin prescribing among postmenopausal women and the elderly is needed; prescribing to these groups may call for special considerations; and more routine reassessments of the appropriateness of long-term preventive treatments among the elderly may be warranted.

\section{Approval of data access}

Access to data was provided and secured through collaboration between the University of Copenhagen and Statistics Denmark. Approval was obtained from the Danish Data Protection Agency through Statistics Denmark. No person 
identifiers were provided to the researchers. According to Danish law, purely registry-based studies do not require ethical approval [38].

\section{Competing Interests}

All authors have completed the Unified Competing Interest form at www.icmje.org/coi disclosure.pdf (available on request from the corresponding author) and declare: no support from any organisation for the submitted work; no financial relationships with any organisations that might have an interest in the submitted work in the previous 3 years; no other relationships or activities that could appear to have influenced the submitted work.

\section{Funding}

No external funding has supported the study 
Figure legends

Figure 1

Prevalence of filled statin prescriptions* by gender and age during 2000-2010

Figure 2

Mean incidence-rate of statin use during 2005-2009 by indication (MI, IHD, stroke and PAD) ${ }^{1}$ according to age and gender

- compared to treatment point prevalence for the same indications in 2010.

Figure 3

Mean incidence-rate of statin use during 2005-2009 by indication (PAC, diabetes, hypertension and no diagnosis) ${ }^{1}$ according to age and gender

- compared to the treatment point prevalence in $\mathbf{2 0 1 0}$ for the same indications

Figure 3

The indication-specific fraction (\%) of incident (A) and prevalent statin users (B)

according to gender and age

\section{Reference List}

1. Randomised trial of cholesterol lowering in $\mathbf{4 4 4 4}$ patients with coronary heart disease: the Scandinavian Simvastatin Survival Study (4S) (1994 Lancet. 344(8934): 1383-1389.

2. Ong HT (2005). The statin studies: from targeting hypercholesterolaemia to targeting the high-risk patient. QJM. 98(8): 599-614.

3. Graham I, Atar D, Borch-Johnsen K, Boysen G, Burell G, Cifkova R et al. (2007). European guidelines on cardiovascular disease prevention in clinical practice: executive summary: Fourth Joint Task Force of the European Society of Cardiology and Other Societies on Cardiovascular Disease Prevention in Clinical Practice (Constituted by representatives of nine societies and by invited experts). Eur Heart J. 28(19): 2375-2414.

4. Christensen, B., Færgemann, O, Heebøll-Nielsen, N, Lous, J, Madsen, L. D, and Stender, S.Forebyggelse af iskæmisk hjertekarsygdom i almen praksis. En klinisk vejledning (3) [Prevention of ischemic cardiovascular disease in general practice. A clinical guideline (3).]. Denmark: The Danish College of General Practitioners (DSAM) 2007

5. Ebrahim S, Casas JP (2012). Statins for all by the age of 50 years? Lancet. 380(9841): 545-547.

6. Goldacre B, Smeeth L (2014). Mass treatment with statins. BMJ. 349: g4745.

7. Jackson PR, Wallis EJ, Haq IU, Ramsay LE (2001). Statins for primary prevention: at what coronary risk is safety assured? Br J Clin Pharmacol. 52(4): 439-446.

8. Clark LT (2003). Treating dyslipidemia with statins: the risk-benefit profile. Am Heart J. 145(3): 387-396.

9. Noaman S, Ibrahim JE, Grenfell R (2014). Prescribing statins for cardiovascular disease prevention in the old: an absence of evidence and an absence of guidelines. Heart Lung Circ. 23(7): 619-624.

10. Abramson J, Wright JM (2007). Are lipid-lowering guidelines evidence-based? Lancet. 369(9557): 168-169.

11. van Staa TP, Smeeth L, Ng ES, Goldacre B, Gulliford M (2013). The efficiency of cardiovascular risk assessment: do the right patients get statin treatment? Heart. 99(21): 1597-1602. 
12. Desai H, Hollingsworth PW, Chugh AR (2015). Statins and Aspirin: Do They Really Work in Women? Am J Cardiovasc Drugs. 15(3): 151-162.

13. Birge SJ (2012). Statins for the primary prevention of cardiovascular disease in women? Menopause. 19(12): 1287-1288.

14. Brugts JJ, Deckers JW (2010). Statin prescription in men and women at cardiovascular risk: to whom and when? Curr Opin Cardiol. 25(4): 484-489.

15. Petursson H, Sigurdsson JA, Bengtsson C, Nilsen TI, Getz L (2012). Is the use of cholesterol in mortality risk algorithms in clinical guidelines valid? Ten years prospective data from the Norwegian HUNT 2 study. J Eval Clin Pract. 18(1): 159-168.

16. Petersen LK, Christensen K, Kragstrup J (2010). Lipid-lowering treatment to the end? A review of observational studies and RCTs on cholesterol and mortality in 80+-year olds. Age Ageing. 39(6): 674-680.

17. Roberts CG, Guallar E, Rodriguez A (2007). Efficacy and safety of statin monotherapy in older adults: a meta-analysis. J Gerontol A Biol Sci Med Sci. 62(8): 879-887.

18. Manuel DG, Lim J, Tanuseputro P, Anderson GM, Alter DA, Laupacis A et al. (2006). Revisiting Rose: strategies for reducing coronary heart disease. BMJ. 332(7542): 659-662.

19. Cook NR, Ridker PM (2014). Further insight into the cardiovascular risk calculator: the roles of statins, revascularizations, and underascertainment in the Women's Health Study. JAMA Intern Med. 174(12): 1964-1971.

20. Petursson H, Getz L, Sigurdsson JA, Hetlevik I (2009). Can individuals with a significant risk for cardiovascular disease be adequately identified by combination of several risk factors? Modelling study based on the Norwegian HUNT 2 population. J Eval Clin Pract. 15(1): 103-109.

21. Mora S, Glynn RJ, Hsia J, Macfadyen JG, Genest J, Ridker PM (2010). Statins for the primary prevention of cardiovascular events in women with elevated high-sensitivity C-reactive protein or dyslipidemia: results from the Justification for the Use of Statins in Prevention: An Intervention Trial Evaluating Rosuvastatin (JUPITER) and meta-analysis of women from primary prevention trials. Circulation. 121(9): 1069-1077.

22. Efficacy and safety of LDL-lowering therapy among men and women: meta-analysis of individual data from 174000 participants in 27 randomised trials (2015 Lancet. 385(9976): 1397-1405.

23. Ray KK, Seshasai SR, Erqou S, Sever P, Jukema JW, Ford I et al. (2010). Statins and all-cause mortality in high-risk primary prevention: a meta-analysis of 11 randomized controlled trials involving 65,229 participants. Arch Intern Med. 170(12): 1024-1031.

24. Taylor F, Huffman MD, Macedo AF, Moore TH, Burke M, Davey SG et al. (2013). Statins for the primary prevention of cardiovascular disease. Cochrane Database Syst Rev. 1: CD004816.

25. van der Harst P, de Boer RA (2010). Statins in the treatment of heart failure. Circ Heart Fail. 3(3): $462-464$.

26. Rysz J, Gluba-Brzozka A, Banach $M$, Wiecek A (2015). Should we use statins in all patients with chronic kidney disease without dialysis therapy? The current state of knowledge. Int Urol Nephrol. 47(5): 805-813.

27. Petretta $M$, Costanzo P, Perrone-Filardi $P$, Chiariello $M$ (2010). Impact of gender in primary prevention of coronary heart disease with statin therapy: a meta-analysis. Int J Cardiol. 138(1): 25-31.

28. Bukkapatnam RN, Gabler NB, Lewis WR (2010). Statins for primary prevention of cardiovascular mortality in women: a systematic review and meta-analysis. Prev Cardiol. 13(2): 84-90.

29. Walsh JM, Pignone M (2004). Drug treatment of hyperlipidemia in women. JAMA. 291(18): 2243-2252. 
30. Ali R, Alexander KP (2007). Statins for the primary prevention of cardiovascular events in older adults: a review of the evidence. Am J Geriatr Pharmacother. 5(1): 52-63.

31. Golomb BA, Evans MA, Dimsdale JE, White HL (2012). Effects of statins on energy and fatigue with exertion: results from a randomized controlled trial. Arch Intern Med. 172(15): 1180-1182.

32. Ko DT, Mamdani M, Alter DA (2004). Lipid-lowering therapy with statins in high-risk elderly patients: the treatment-risk paradox. JAMA. 291(15): 1864-1870.

33. Upmeier E, Korhonen MJ, Helin-Salmivaara A, Huupponen R (2012). Statin use among older Finns stratified according to cardiovascular risk. Eur J Clin Pharmacol.

34. Sheppard JP, Singh S, Fletcher K, McManus RJ, Mant J (2012). Impact of age and sex on primary preventive treatment for cardiovascular disease in the West Midlands, UK: cross sectional study. BMJ. 345: e4535.

35. Wallach-Kildemoes $H$, Diderichsen $F$, Krasnik A, Lange $T$, Andersen $M$ (2012). Is the high-risk strategy to prevent cardiovascular disease equitable? A pharmacoepidemiological cohort study. BMC Public Health. 12(1): 610 .

36. Wallach-Kildemoes $\mathrm{H}$, Hendriksen C, Andersen M (2012). Drug utilization according to reason for prescribing: a pharmacoepidemiologic method based on an indication hierarchy. Pharmacoepidemiol Drug Saf. 21(10): 1027-1035.

37. Wallach-Kildemoes $\mathrm{H}$, Vass $\mathrm{M}$, Hendriksen $\mathrm{C}$, Andersen $\mathrm{M}$ (2012). Statin utilization according to indication and age: A Danish cohort study on changing prescribing and purchasing behaviour. Health Policy(108): 216-227.

38. Thygesen LC, Daasnes C, Thaulow I, Bronnum-Hansen H (2011). Introduction to Danish (nationwide) registers on health and social issues: Structure, access, legislation and archiving. Scand J Public Health. 39(Supple 7): 12-16.

39. Kildemoes HW, Sorensen HT, Hallas J (2011). The Danish National Prescription Registry. Scand J Public Health. 39(Supple 7): 38-41.

40. WHO Collaborating centre for Drug Statistics Methodology, Guidelines for ATC classification and DDD assignment, 2014. Oslo: Norwegian Institute of Public Health 2014

41. Lynge E, Sandegaard JL, Rebolj M (2011). The Danish National Patient Register. Scand J Public Health. 39(7 Suppl): 30-33.

42. Jensen HK (2002). The molecular genetic basis and diagnosis of familial hypercholesterolemia in Denmark. Dan Med Bull. 49(4): 318-345.

43. DeWilde S, Carey IM, Bremner SA, Richards N, Hilton SR, Cook DG (2003). Evolution of statin prescribing 1994-2001: a case of agism but not of sexism? Heart. 89(4): 417-421.

44. Chokshi NP, Messerli FH, Sutin D, Supariwala AA, Shah NR (2012). Appropriateness of statins in patients aged $>/=80$ years and comparison to other age groups. Am J Cardiol. 110(10): 1477-1481.

45. Kildemoes HW, Stovring H, Andersen M (2008). Driving forces behind increasing cardiovascular drug utilization: a dynamic pharmacoepidemiological model. Br J Clin Pharmacol. 66(6): 885-895.

46. Cho L, Hoogwerf B, Huang J, Brennan DM, Hazen SL (2008). Gender differences in utilization of effective cardiovascular secondary prevention: a Cleveland clinic prevention database study. J Womens Health (Larchmt ). 17(4): 515-521. 
47. Tonstad S, Rosvold EO, Furu K, Skurtveit S (2004). Undertreatment and overtreatment with statins: the Oslo Health Study 2000-2001. J Intern Med. 255(4): 494-502.

48. Rasmussen JN, Gislason GH, Abildstrom SZ, Rasmussen S, Gustafsson I, Buch P et al. (2005). Statin use after acute myocardial infarction: a nationwide study in Denmark. Br J Clin Pharmacol. 60(2): 150-158.

49. Ni CD, Asplund K, Asberg S, Callaly E, Cuadrado-Godia E, Diez-Tejedor E et al. (2013). Statin therapy and outcome after ischemic stroke: systematic review and meta-analysis of observational studies and randomized trials. Stroke. 44(2): 448-456.

50. Shepherd J, Blauw GJ, Murphy MB, Bollen EL, Buckley BM, Cobbe SM et al. (2002). Pravastatin in elderly individuals at risk of vascular disease (PROSPER): a randomised controlled trial. Lancet. 360(9346): 16231630.

51. Tziomalos K, Athyros VG, Karagiannis A, Mikhailidis DP (2009). Dyslipidemia as a risk factor for ischemic stroke. Curr Top Med Chem. 9(14): 1291-1297.

52. NHS Health Check. 2015. 2015.

53. QRISK 2-2014 cardiovascular disease risk calculator. 2014.

54. Abramson JD, Rosenberg HG, Jewell N, Wright JM (2013). Should people at low risk of cardiovascular disease take a statin? BMJ. 347: $\mathbf{6 6 1 2 3 .}$

55. Taylor F, Ward K, Moore TH, Burke M, Davey SG, Casas JP et al. (2011). Statins for the primary prevention of cardiovascular disease. Cochrane Database Syst Rev(1): CD004816.

56. Kostis WJ, Cheng JQ, Dobrzynski JM, Cabrera J, Kostis JB (2012). Meta-analysis of statin effects in women versus men. J Am Coll Cardiol. 59(6): 572-582.

57. Hirsch AT, Haskal ZJ, Hertzer NR, Bakal CW, Creager MA, Halperin JL et al. (2006). ACC/AHA Guidelines for the Management of Patients with Peripheral Arterial Disease (lower extremity, renal, mesenteric, and abdominal aortic). J Vasc Interv Radiol. 17(9): 1383-1397.

58. Randomized trial of the effects of cholesterol-lowering with simvastatin on peripheral vascular and other major vascular outcomes in 20,536 people with peripheral arterial disease and other high-risk conditions (2007 J Vasc Surg. 45(4): 645-654.

59. Collins R, Armitage J, Parish S, Sleigh P, Peto R (2003). MRC/BHF Heart Protection Study of cholesterollowering with simvastatin in 5963 people with diabetes: a randomised placebo-controlled trial. Lancet. 361(9374): 2005-2016.

60. Collins R, Armitage J, Parish S, Sleight P, Peto R (2004). Effects of cholesterol-lowering with simvastatin on stroke and other major vascular events in 20536 people with cerebrovascular disease or other high-risk conditions. Lancet. 363(9411): 757-767.

61. Stein LH, Berger J, Tranquilli M, Elefteraides JA (2013). Effect of statin drugs on thoracic aortic aneurysms. Am J Cardiol. 112(8): 1240-1245.

62. Celik T, Demirkol S (2011). Statin dilemma in the management of chronic heart failure. Int J Cardiol. 147(3): 452-453.

63. Manuel DG, Kwong K, Tanuseputro P, Lim J, Mustard CA, Anderson GM et al. (2006). Effectiveness and efficiency of different guidelines on statin treatment for preventing deaths from coronary heart disease: modelling study. BMJ. 332(7555): 1419. 
64. Conroy RM, Pyorala K, Fitzgerald AP, Sans S, Menotti A, De Backer G et al. (2003). Estimation of ten-year risk of fatal cardiovascular disease in Europe: the SCORE project. Eur Heart J. 24(11): 987-1003.

65. Lindman AS, Veierod MB, Pedersen JI, Tverdal A, Njolstad I, Selmer R (2007). The ability of the SCORE highrisk model to predict 10-year cardiovascular disease mortality in Norway. Eur J Cardiovasc Prev Rehabil. 14(4): 501-507.

66. Stone NJ, Robinson J, Lichtenstein AH, Bairey Merz CN, Lloyd-Jones DM, Blum CB et al. (2013). 2013 ACC/AHA Guideline on the Treatment of Blood Cholesterol to Reduce Atherosclerotic Cardiovascular Risk in Adults: A Report of the American College of Cardiology/American Heart Association Task Force on Practice Guidelines. J Am Coll Cardiol.

67. Ridker PM, Cook NR (2013). Statins: new American guidelines for prevention of cardiovascular disease. Lancet. 382(9907): 1762-1765.

68. loannidis JP (2014). More than a billion people taking statins?: Potential implications of the new cardiovascular guidelines. JAMA. 311(5): 463-464.

69. Kaplan RM, Ong M (2007). Rationale and public health implications of changing CHD risk factor definitions. Annu Rev Public Health. 28: 321-344.

70. Tuikkala P, Hartikainen S, Korhonen MJ, Lavikainen P, Kettunen R, Sulkava R et al. (2010). Serum total cholesterol levels and all-cause mortality in a home-dwelling elderly population: a six-year follow-up. Scand J Prim Health Care. 28(2): 121-127.

71. Rabar S, Harker M, O'Flynn N, Wierzbicki AS (2014). Lipid modification and cardiovascular risk assessment for the primary and secondary prevention of cardiovascular disease: summary of updated NICE guidance. BMJ. 349: g4356.

72. Perk J, De BG, Gohlke H, Graham I, Reiner Z, Verschuren M et al. (2012). European Guidelines on cardiovascular disease prevention in clinical practice (version 2012). The Fifth Joint Task Force of the European Society of Cardiology and Other Societies on Cardiovascular Disease Prevention in Clinical Practice (constituted by representatives of nine societies and by invited experts). Eur Heart J. 33(13): 16351701.

73. Engebretsen E, Vollestad NK, Wahl AK, Robinson HS, Heggen K (2015). Unpacking the process of interpretation in evidence-based decision making. J Eval Clin Pract. 21(3): 529-531.

74. Mihaylova B, Emberson J, Blackwell L, Keech A, Simes J, Barnes EH et al. (2012). The effects of lowering LDL cholesterol with statin therapy in people at low risk of vascular disease: meta-analysis of individual data from 27 randomised trials. Lancet. 380(9841): 581-590.

75. Mosca L (2015). Sex, statins, and statistics. Lancet. 385(9976): 1368-1369.

76. Savarese G, Gotto AM, Jr., Paolillo S, D'Amore C, Losco T, Musella F et al. (2013). Benefits of statins in elderly subjects without established cardiovascular disease: a meta-analysis. J Am Coll Cardiol. 62(22): 2090-2099.

77. Wang W, Zhang B (2014). Statins for the prevention of stroke: a meta-analysis of randomized controlled trials. PLoS One. 9(3): e92388.

78. Shepherd J (2004). A prospective study of pravastatin in the elderly at risk: new hope for older persons. Am J Geriatr Cardiol. 13(3 Suppl 1): 17-24.

79. Cham S, Evans MA, Denenberg JO, Golomb BA (2010). Statin-associated muscle-related adverse effects: a case series of 354 patients. Pharmacotherapy. 30(6): 541-553. 
80. Auer J, Sinzinger H, Franklin B, Berent R (2014). Muscle- and skeletal-related side-effects of statins: tip of the iceberg? Eur J Prev Cardiol.

81. Golomb BA (2014). Misinterpretation of trial evidence on statin adverse effects may harm patients. Eur J Prev Cardiol.

82. Ruscica M, Macchi C, Morlotti B, Sirtori CR, Magni P (2014). Statin therapy and related risk of new-onset type 2 diabetes mellitus. Eur J Intern Med.

83. Taylor BA, Thompson PD (2015). Muscle-related side-effects of statins: from mechanisms to evidencebased solutions. Curr Opin Lipidol. 26(3): 221-227.

84. Deichmann R, Lavie C, Andrews S (2010). Coenzyme q10 and statin-induced mitochondrial dysfunction. Ochsner J. 10(1): 16-21.

85. Larsen S, Stride N, Hey-Mogensen M, Hansen CN, Bang LE, Bundgaard H et al. (2013). Simvastatin effects on skeletal muscle: relation to decreased mitochondrial function and glucose intolerance. J Am Coll Cardiol. 61(1): 44-53.

86. Culver AL, Ockene IS, Balasubramanian R, Olendzki BC, Sepavich DM, Wactawski-Wende J et al. (2012). Statin use and risk of diabetes mellitus in postmenopausal women in the Women's Health Initiative. Arch Intern Med. 172(2): 144-152.

87. Bhardwaj S, Selvarajah S, Schneider EB (2013). Muscular effects of statins in the elderly female: a review. Clin Interv Aging. 8: 47-59.

88. Robinson JG, Booth B (2010). Statin use and lipid levels in older adults: National Health and Nutrition Examination Survey, 2001 to 2006. J Clin Lipidol. 4(6): 483-490.

89. Hamer M, Lavoie KL, Bacon SL (2014). Taking up physical activity in later life and healthy ageing: the English longitudinal study of ageing. Br J Sports Med. 48(3): 239-243.

90. Wei MY, Ito MK, Cohen JD, Brinton EA, Jacobson TA (2013). Predictors of statin adherence, switching, and discontinuation in the USAGE survey: understanding the use of statins in America and gaps in patient education. J Clin Lipidol. 7(5): 472-483.

91. Lee KT, Lai WT, Chu CS, Tsai LY, Yen HW, Voon WC et al. (2004). Effect of withdrawal of statin on C-reactive protein. Cardiology. 102(3): 166-170.

92. Tziomalos K, Athyros VG, Mikhailidis DP (2008). Statin discontinuation: an underestimated risk? Curr Med Res Opin. 24(11): 3059-3062.

93. Li JJ (2011). Inflammatory rebound phenomenon after abrupt withdrawal of statin is a mature point of view but not hypotheses. Int J Cardiol. 151(1): 120.

94. Tehrani FR, Behboudi-Gandevani S, Ghanbarian A, Azizi F (2014). Effect of menopause on cardiovascular disease and its risk factors: a 9-year follow-up study. Climacteric. 17(2): 164-172.

95. Rose G (1985). Sick individuals and sick populations. Int J Epidemiol. 14(1): 32-38.

96. Mangin D, Sweeney K, Heath I (2007). Preventive health care in elderly people needs rethinking. BMJ. 335(7614): 285-287. 